\title{
Transforaminal Percutaneous Endoscopic Discectomy in Parkinson Disease: Preliminary Results and Short Review of the Literature
}

\author{
Stylianos Kapetanakis ${ }^{1}$, Eirini Giovannopoulou ${ }^{1}$, Triphonas Thomaidis ${ }^{1}$, George Charitoudis ${ }^{1}$, \\ Pavlos Pavlidis ${ }^{3}$, Konstantinos Kazakos ${ }^{2}$ \\ ${ }^{l}$ Spine Department and Deformities, Interbalkan European Medical Center, Thessaloniki, \\ ${ }^{2}$ Department of Orthopaedic Surgery, University Hospital of Alexandroupolis, Medical School, Democritus University of \\ Thrace, Alexandroupolis, \\ ${ }^{3}$ Laboratory of Forensic Sciences, Medical School, Democritus University of Thrace, Alexandroupolis, Greece
}

\begin{abstract}
Objective: To study the effectiveness of Transforaminal Percutaneous Endoscopic Discectomy (TPED) for lumbar disc herniation in patients with Parkinson disease (PD).

Methods: Fifteen patients diagnosed with PD and lumbar disc hernia were recruited to the study. All patients underwent TPED. Mean age was $61.27 \pm 6$ years, with 8 male (53.3\%) and 7 female patients (46.7\%). Level of operation was L3-4 (33.3\%), L4-5 (33.3\%) and L5-S1 (33.3\%). Visual analogue scale (VAS) for leg pain and Oswestry Disabillity Index (ODI) for back pain, as well as the Medical Outcomes Study Questionnaire Short-Form 36 Health Survey (SF-36) for health-related quality of life (HRQLL) were assessed right before surgery and at 6 weeks, 3,6 , and 12 months after surgery.

Results: VAS and ODI showed significant $(p<0.005)$ reduction one year after TPED, with a percentage improvement of $83.9 \%$ and $79.4 \%$, respectively. Similarly, all aspects of quality of life (SF-36) were significantly $(p<0.005)$ improved 1 year after the procedure. Bodily pain and role physical demonstrated the highest increase followed by role emotional, physical function, social function, vitality, mental health, and general health. Beneficial impact of TPED on clinical outcome and HRQoL was independent of gender and operated level.

Conclusion: TPED is effective in reducing lower limb symptoms and low back pain in patients with lumbar disc hernia, suffering from PD. Positive effect of endoscopy is, also, evident in HRQoL of those patients one year after the procedure.
\end{abstract}

Key Words: Parkinson disease $\cdot$ Endoscopy $\cdot$ Percutaneous discectomy $\cdot$ Quality of life

\section{INTRODUCTION}

Parkinson disease (PD) is a degenerative disorder of the basal ganglions, pathophysiologically characterized by the loss of dopaminergic neurons in the area of substantia nigra pars compacta that leads mainly to motor dysfunstion ${ }^{4,21}$. Clinical manifestation includes four main or "cardinal" symptoms: akinesia or bradykinesia, rest tremor, rigidity and postural instability ${ }^{4,21)}$. PD is a disease of the aged population with a peak incidence at the 8 th decade of life ${ }^{32}$. Prevalence is estimated between 65.6 per 100,000 to 12,500 per 100,000 in different European studies. Annually, there are about 5 to 346 new cases

- Received: July 27, 2016 • Revised: September 8, 2016

- Accepted: September 13, 2016

Corresponding Author: Kapetanakis Stylianos

Spine Department and Deformities, Interbalkan European Medical

Center, Thessaloniki, PC 57001, Greece

Tel: +30-6972707384, Fax: +30-23112-89109

E-mail: stkapetanakis@yahoo.gr

@ This is an open access article distributed under the terms of the Creative Commons Attribution Non-Commercial License (http://creativecommons.org/licenses/by-nc/4.0/) which permits unrestricted non-commercial use, distribution, and reproduction in any medium, provided the original work is properly cited. per 100,000 and this number is expected to rise as the population ages ${ }^{34)}$.

PD is associated with spine deformity and pain ${ }^{10,13,14)}$. PD patients suffer frequently from non-specific low back pain ${ }^{5)}$. Origins of the pain are muscles, paraspinal soft tissues and bones. Mechanism of pain is abnormal stress distribution to those structures through muscle rigidity and postural deformity of the spine. Nevertheless, stooped posture of the trunk with mildly bent hips and knees alters spine biomechanics, transpositions the center of gravity and exaggerates force distribution, especially on the ventral elements of vertebrae and discs ${ }^{5}$. Impaired biomechanics act synergically with aging and lead to profound degeneration of the intervertebral discs, hernia formation and radiculopathy ${ }^{1)}$. Background of the disease explains the high prevalence and intensity of low back pain and radiculopathy in this population, independently from the duration and the severity of the disease or the "off phases" surgery is frequently indicated in patients diagnosed with PD to preserve functional ability. Open spine surgery, however, has poor clinical outcome with high complication and revision rates, especially due to spinal instability ${ }^{2,2029)}$.

Lumbar discectomy is a common procedure for spinal sur- 
geons and minimally invasive techniques are becoming more popular. Transforaminal percutaneous endoscopic discectomy (TPED) is a posterolateral, endoscopic technique that access the disc pathology through the intervertebral foramen and the anatomic triangle, described by Kambin and Brager ${ }^{177}$. Endoscopy is associated with reduced hospital stay, lower postoperative morbidity and shorter rehabilitation period ${ }^{22,28)}$. Transforaminal approach is atraumatic for the dorsal structures (muscles, bones, ligaments) and minimizes the scaring of the epidural space ${ }^{28)}$. The latter effect is of substantial importance on revision surgery.

Therefore, patients suffering from PD and lumbar disc hernia are high risk population for open procedures and may benefit from the advantages of endoscopic management of their hernia. Our intention is to investigate the clinical outcome and the progress of health-related quality of life (HRQoL) one year after TPED in PD patients with lumbar disc hernia.

\section{MATERIALS ANS METHODS}

\section{Patients}

Fifteen patients diagnosed with PD and lumbar disc hernia were enrolled in the study and scheduled to undergo TPED. All patients agreed to participate in the study and provided informed written consent. The ptotocol was approved by the Medical Council of Interbalkan European Medical Center and the Local Ethics Committee.

\section{1) Inclusion criteria}

(1) Previous diagnosis of PD according to UK Parkinson's Disease Society Brain Bank clinical diagnostic criteria ${ }^{16}$, (2) radiculopathy, (3) positive nerve root tension sign, (4) sensory or motor neurologic deficit, (5) hernia confirmed by magnetic resonance imaging of the lumbar spine, in compliance with clinical findings, (6) failure of 12 -week conservative treatment.

\section{2) Exclusion criteria}

(1) Noncontaminated or sequestrated disc hernia, (2) central or lateral recess stenosis, (3) recurrent disc hernia, (4) previous spine surgery, (5) concomitant conditions, such as spondylolisthesis or degenerative scoliosis, (6) vertebral fracture (mainly osteoporotic), (7) vertebral infection or tumor, (8) previous deep brain stimulation surgery.

\section{Methods}

All patients that fulfilled the aforementioned criteria were recruited to the study and underwent lumbar endoscopic discectomy. The surgical technique was TPED, performed at the same center and by the same experienced spine surgeon. Perioperative complications were not observed.
Follow-up period was 1 year. Visual Analogue Scale (VAS) for leg pain and Oswestry Disability Index (ODI) for back pain were utilized for clinical outcome assessment. The Medical Outcomes Study Questionnaire Short-Form 36 Health Survey (SF-36) was used for the assessment of HRQoL.

\section{VAS for Leg Pain}

Personal score was measured in millimeters ( $\mathrm{mm}$ ), giving an objective perception over the patients' radicular symptomato$\operatorname{logy}^{37}$. Minimum clinical significant change is associated with a change of at least $16 \mathrm{~mm}$ in VAS for leg pain, in lumbar spine interventions ${ }^{7}$. VAS has been previously used in the literature in PD for the assessment of pain, which is a common condition among the patients, ${ }^{5,11,33)}$.

\section{Oswestry Disability Index}

ODI is a condition-specific measure for the evaluation of low back pain in daily living. It is commonly used in the outcome assessment of spine surgery, as it is easy and reliable ${ }^{8,12}$. The interviewer asked the patients to choose the statement that was best compatible with their clinical condition. Its individual's total score was expressed as percentage and represented the percentage of disability experienced in everyday life due to low back problems. Minimum clinical significant change comprises a change in ODI of at least 12.8 points at the percentage scale, in patients undergoing lumbar spine surgery ${ }^{7}$.

\section{The Medical Outcomes Study Questionnaire Short-Form 36 Health Survey}

The SF-36 is the most commonly used generic HRQoL in the clinical assessment of spine surgery ${ }^{38)}$. It consists by multiitem scales focused on eight different aspects of general health; physical function (PF), role limitations due to physical problems (RP), bodily pain (BP), general health $(\mathrm{GH})$ perceptions, vitality, energy, and fatigue (V), social function (SF), role limitations due to emotional problems (RE), and mental health (MH). The interviewer assisted the patients to enter the required data, which were then coded, summed and transformed to a percentage scale. Generally, the higher the score is the better the health status of the patient ${ }^{36}$.

Insofar, PD affects HRQoL, patient-oriented questionnaires are broadly applied in those patients, in order to investigate the disease's natural course and responsiveness to treatment $^{30)}$. PD-specific measures of quality of life have been developed, such as the 39-item Parkinson Disease Questionnaire or the Parkinson Disease Quality of Life Measure ${ }^{9,26}$. However, in our study we used SF-36, a generic HRQoL applied in the general population. SF-36 has been tested for its validity, reliability and responsiveness in different conditions, including spinal disorders and PD ${ }^{3,15,27,30,35)}$. Nevertheless, SF-36 is widely accepted as outcome measure in spine surgery and 
normative data exist in the literature, facilitating comparison with traditional open procedures and general population.

All data (VAS, ODI, SF-36) were collected by interview, in order to ensure optimal comprehension, considering the effect of PD in cognitive ability. The assessment took place right before surgery and was repeated at specific intervals during the follow-up period (6 weeks, 3 months, 6 months, 12 months).

\section{Surgical Technique}

The procedure was TPED, performed under local anesthesia and mild sedation, by the same experienced in the technique spinal surgeon. Patients positioning was lateral decubitus, for optimal enlargement of the foraminal space. The surgical field was disinfected and local anesthesia was applied at the needle entry point. Subsequently, the needle was promoted $11 \mathrm{~cm}$ laterally of the midline and through the anatomic triangle of Kambin, in order to access the foramen. The procedure took place under fluoroscopic guidance. The position of the needle was confirmed and, then, mild sedation and anal-

Table 1. Patients' demographic characteristics

\begin{tabular}{lc}
\hline \hline Variable & Value \\
\hline Age $(\mathrm{yr})$ & $61.27 \pm 6.0$ \\
Sex & \\
$\quad$ Male & $8(53.3)$ \\
Female & $7(46.7)$ \\
Level of operation & \\
L3-L4 & $5(33.3)$ \\
L4-L5 & $5(33.3)$ \\
L5-S1 & $5(33.3)$ \\
\hline
\end{tabular}

Values are presented as mean \pm standard deviation or number (\%). gesia were provided with Fentanyl (Fentanyl ampule), to facilitate the passage of the reamers. Three different reamers were sequentially passed, in ascending order from 5.5 to $6.5 \mathrm{~mm}$ and, finally, to $7.5-\mathrm{mm}$ diameter (Joimax System). The cannula and the endoscope were introduced in place and the herniated material was removed by graspers. No perioperative complications were observed. The patients were monitored for the first postoperative hour in the wards and, then, mobilized. Finally, all of them were discharged within 24 hours.

\section{Statistical Analysis}

Collected data were analyzed with the use of SPSS ver. 13.0 (SPSS Inc., Chicago, IL, USA). Continuous variables (age, VAS, ODI, SF-36 components) are expressed as mean \pm standard deviation and categorical variables (sex, level of operation) as percentages. The Kolmogorov-Smirnov test was utilized for normality analysis.

Student t-test and Wilcoxon test were utilized for the comparison of the quantitive-continuous variables (VAS, ODI, SF36 components) in dependent samples at each time point, for normal or not distribution, respectively.

One-way analysis of variance and Kruskal-Wallis tests were applied for the comparison between independent samples according to sex (2 samples, male/female), for normal or not distribution, respectively.

Student t-test and Mann-Whitney tests were used for the comparison between independent samples according to level of operation (3 samples, L3-4/L4-5/L5-S1), for normal and not distribution respectively.

\section{RESULTS}

Total number of patients was fifteen. Demographic charac-

Table 2. VAS, ODI, and SF-36 components at baseline and at 6 weeks, 3 months, 6 months and 12 months postsurgery

\begin{tabular}{llllll}
\hline \hline Measure & Baseline. & \multicolumn{1}{c}{6 Weeks } & \multicolumn{1}{c}{3 Months } & \multicolumn{1}{c}{6 Months } & 12 Months \\
\hline VAS (mm) & $65.7 \pm 11.6$ & $31.5 \pm 7.0\left(<0.001^{*}\right)$ & $20.0 \pm 4.5\left(<0.001^{*}\right)$ & $12.3 \pm 2.3\left(<0.001^{*}\right)$ & $10.2 \pm 2.1\left(0.003^{*}\right)$ \\
ODI (\%) & $58.8 \pm 13.1$ & $19.8 \pm 5.3\left(<0.001^{*}\right)$ & $14.4 \pm 3.5\left(<0.001^{*}\right)$ & $10.3 \pm 2.9\left(0.003^{*}\right)$ & $11.3 \pm 5.3(0.379)$ \\
PF (\%) & $31.8 \pm 3.6$ & $45.1 \pm 7.2\left(<0.001^{*}\right)$ & $49.9 \pm 6.7\left(0.001^{*}\right)$ & $52.6 \pm 7.1\left(0.001^{*}\right)$ & $54.0 \pm 8.1\left(0.131^{*}\right)$ \\
RP (\%) & $11.9 \pm 3.4$ & $30.4 \pm 3.4\left(<0.001^{*}\right)$ & $34.5 \pm 4.6\left(<0.001^{*}\right)$ & $40.9 \pm 5.0\left(<0.001^{*}\right)$ & $43.0 \pm 5.2\left(0.002^{*}\right)$ \\
BP (\%) & $27.3 \pm 4.2$ & $50.4 \pm 5.2\left(<0.001^{*}\right)$ & $56.1 \pm 5.9\left(<0.001^{*}\right)$ & $62.2 \pm 5.2\left(<0.001^{*}\right)$ & $64.7 \pm 6.0\left(<0.001^{* *}\right)$ \\
GH (\%) & $41.1 \pm 4.6$ & $42.9 \pm 3.7\left(<0.001^{*}\right)$ & $44.9 \pm 4.3\left(0.003^{*}\right)$ & $48.1 \pm 4.7\left(0.002^{*}\right)$ & $50.4 \pm 4.4\left(0.001^{*}\right)$ \\
V (\%) & $33.1 \pm 3.5$ & $39.8 \pm 4.8\left(<0.001^{*}\right)$ & $43.5 \pm 4.7\left(<0.001^{*}\right)$ & $46.9 \pm 6.3\left(0.002^{*}\right)$ & $47.9 \pm 6.5\left(0.048^{*}\right)$ \\
SF (\%) & $45.1 \pm 6.7$ & $54.2 \pm 6.5\left(<0.001^{*}\right)$ & $58.5 \pm 5.9\left(<0.001^{*}\right)$ & $61.8 \pm 5.1\left(<0.001^{*}\right)$ & $63.7 \pm 5.8\left(0.001^{*}\right)$ \\
RE (\%) & $44.6 \pm 5.9$ & $58.1 \pm 6.1\left(<0.001^{*}\right)$ & $62.1 \pm 6.2\left(<0.001^{*}\right)$ & $66.7 \pm 5.8\left(<0.001^{*}\right)$ & $67.1 \pm 5.8(0.674)$ \\
MH (\%) & $61.9 \pm 4.7$ & $64.3 \pm 5.2\left(<0.001^{*}\right)$ & $67.7 \pm 6.8\left(0.003^{*}\right)$ & $69.2 \pm 7.5\left(0.002^{*}\right)$ & $71.4 \pm 6.8\left(0.001^{*}\right)$ \\
\hline
\end{tabular}

Values are presented as mean \pm standard deviation (p-value).

VAS, visual analogue scale; ODI, Oswestry Disabillity Index; SF-36, Medical Outcomes Study Short-form Questionnaire; PF, physical function; RP, role physical; BP, bodily pain; GH, general health; V, vitality; SF, social function; RE, role limitations due to emotional problems; MH, mental health.

p $<0.05$, statistically significant. 
teristics of our population are presented at Table 1.

The baseline values and postoperative progress of VAS for leg pain, ODI, and SF-36 components are presented at Table 2. The level of significance is determined at $\mathrm{a}=0.05$ or $\mathrm{p}<0.05$. $\mathrm{p}$-values embedded in the table comprise the comparison of present values with the immediately preceding measurements.

VAS and ODI scores presented significant reduction during the follow-up period. As far as ODI is concerned, the result seemed to stabilize at 6 months postoperatively. Between 6 and 12 months, there was a slight increase in ODI. However, the change remained insignificant $(p=0.379)$. All the aspects of quality of life were gradually improved one year post operatively, in a statistically significant way $(\mathrm{p}<0.005)$. The difference was significant at every time interval $(\mathrm{p}<0.05)$, except for the aspects of PF and RE. PF and RE were stabilized within 6 months and their increase was not significant thereafter $(p=$ 0.131 and $p=0.674$, respectively). Progressive change of ODI, VAS and SF-36 components is described in Fig. 1.

The end-point impact of TPED on clinical condition and quality of life is presented at Table 3. VAS and ODI absolute reduction scores are converted into improvement percentages, while the progress of SF-36 aspects is presented in absolute reduction values. The results were furtherly analyzed for any statistical significant difference according to gender and level of operation, as independent populations. p-values and statistical test utilized are, also, provided at Table 3.

The final outcome does not differentiate according to sex or level of operation, except from VAS score. After the 12month follow-up, the percentage improvement of VAS was the highest for the level L4-L5 and the difference was signifi-

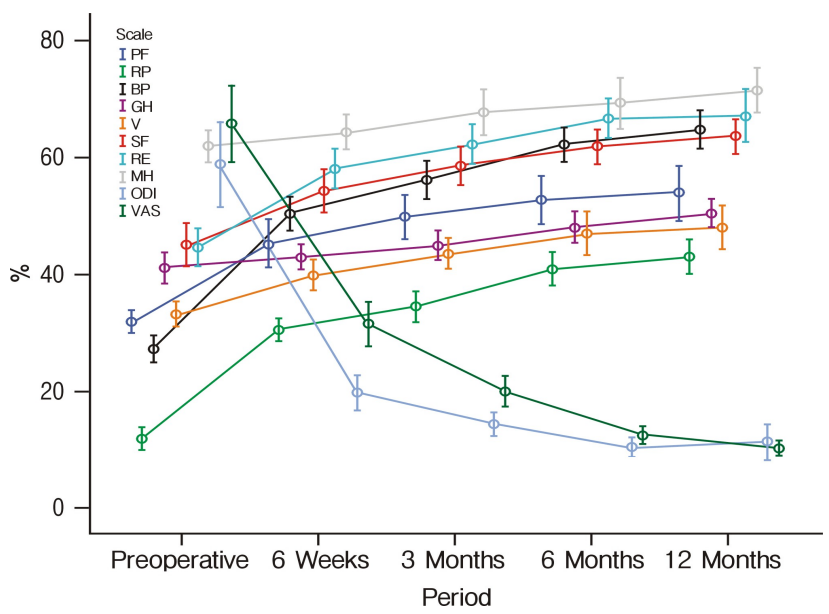

Fig. 1. Progressive change of ODI, VAS, and SF-36 during the follow-up period. Comparative progress of VAS, ODI, and SF36 components is highlighted with different colors, from baseline to 12 months postoperatively. VAS, visual analogue scale; ODI, Oswestry Disabillity Index; SF-36, Medical Outcomes Study Short-form Questionnaire; PF, physical function; RP, role physical; BP, bodily pain; GH, general health; V, vitality; SF, social function; RE, role limitations due to emotional problems; $\mathrm{MH}$, mental health. cant only compared to $\mathrm{L} 5-\mathrm{S} 1(\mathrm{p}=0.032)$.

\section{DISCUSSION}

PD is traditionally described as a disease of motor disorder and spine is commonly affected in the disease's natural course $^{4,10,21)}$. Parkinsonic spine balance is violated on the sagittal and coronal planes. Sagittal deformities include stooped posture of the trunk (leading in extreme cases to camptocormia) and antecollis. Coronal plane disorders mainly refer to Pisa syndrome and scoliosis ${ }^{10)}$. PD induces spinal malalignment and instability, probably proportionate to disease severity ${ }^{6,24)}$. In the thoracolumbar section, the natural curves of thoracic kyphosis and lumbar lordosis are shown to increase in patients with $\mathrm{PD}^{24)}$. Potential pathophysiologic mechanisms include muscle rigidity, flexion dystonia of the trunk, impaired proprioception and kinaesthesia, as well as pharmacotherapy with dopamine agonists. Paraspinal muscle myopathy, poor bone quality and progressive degeneration due to aging act cumulatively to established spinal deformity ${ }^{10}$.

PD alters spine biomechanics and poses limitations to spine surgery. Malalignment of the spino-pelvic parameters, described in PD, predisposes to bad outcome in lumbar spine surgery $^{19,24)}$. Neuromuscular dysfunction is independently increasing the risk of complications ${ }^{20,29)}$. However, few studies have investigated the clinical outcome of spine surgery in patients diagnosed with $\mathrm{PD}^{2,20,29)}$. Existing data converge on a high complication rate, mainly due to instability and hardware failure. Revision surgery is frequently required ${ }^{2,20,29)}$. Moreover, $\mathrm{PD}$ patients experience more frequently common complica-

Table 3. Percentage improvements of VAS, ODI, and absolute change of SF-36 components at the end of 1-year follow-up

\begin{tabular}{lccc}
\hline \hline Measure & $\begin{array}{c}\text { Preoperative- } \\
12 \text { months }\end{array}$ & $\begin{array}{c}\text { p-value } \\
(\text { sex })\end{array}$ & $\begin{array}{c}\text { p-value } \\
\text { (level) }\end{array}$ \\
\hline VAS (\%) & $83.9 \pm 4.8$ & $0.495^{\mathrm{a}}$ & $0.032^{*, c}$ \\
ODI (\%) & $79.4 \pm 11.6$ & $0.074^{\mathrm{a}}$ & $0.507^{\mathrm{c}}$ \\
PF (\%) & $22.2 \pm 8.0$ & $0.119^{\mathrm{a}}$ & $0.069^{\mathrm{c}}$ \\
RP (\%) & $31.1 \pm 5.2$ & $0.126^{\mathrm{a}}$ & $0.161^{\mathrm{c}}$ \\
BP (\%) & $37.4 \pm 5.4$ & $0.320^{\mathrm{a}}$ & $0.532^{\mathrm{c}}$ \\
GH (\%) & $9.3 \pm 3.8$ & $0.828^{\mathrm{a}}$ & $0.334^{\mathrm{c}}$ \\
V (\%) & $14.8 \pm 5.4$ & $0.281^{\mathrm{b}}$ & $0.464^{\mathrm{d}}$ \\
SF (\%) & $18.6 \pm 4.8$ & $0.255^{\mathrm{a}}$ & $0.196^{\mathrm{c}}$ \\
RE (\%) & $22.5 \pm 7.7$ & $0.786^{\mathrm{a}}$ & $0.774^{\mathrm{c}}$ \\
MH (\%) & $9.5 \pm 6.1$ & $0.086^{\mathrm{a}}$ & $0.134^{\mathrm{c}}$ \\
\hline
\end{tabular}

Values are presented as mean \pm standard deviation.

VAS, visual analogue scale; ODI, Oswestry Disabillity Index; SF-36, Medical Outcomes Study Short-form Questionnaire; PF, physical function; RP, role physical; BP, bodily pain; $\mathrm{GH}$, general health; V, vitality; SF, social function; RE, role limitations due to emotional problems; $\mathrm{MH}$, mental health. " $\mathrm{p}<0.05$, statistically significant.

at-test. bann-Whitney. ${ }^{\mathrm{c}}$ One-way analysis of variance.

${ }^{\mathrm{d}}$ Kruskal-Wallis. 
tions, such as postoperative infections ${ }^{2,20)}$.

Our present knowledge is limited and, only, refers to traditional open procedures. The aforementioned studies are characterized by retrospective data selection heterogeneous samples, concerning spine pathology and type of surgery and condition-specific measures and tools that fail to interpret the findings in terms of quality of life. Our study focuses on the effectiveness of TPED in PD patients diagnosed with lumbar disc hernia, with special interest to health related quality of life.

Our findings converge that endoscopic discectomy significantly improves radiculopathy and disability due to low back pain in patients diagnosed with PD and lumbar disc hernia, 1 year after the procedure. Specifically, TPED achieved a mean improvement of $83 \%$ and $79.4 \%$ in VAS and ODI measures, respectively. Nellensteijn et al. ${ }^{23)}$ reviewed the literature to determine the effectiveness of TPED in symptomatic patients with lumbar disc herniation. The procedure produced an improvement ranging from $65 \%$ to $89 \%$ for VAS and $74 \%$ to $90 \%$ for ODI. The results indicate that the clinical outcome of TPED is comparable among patients with and without PD. Kapetanakis et al. ${ }^{18)}$ conducted a case-control study to compare the clinical effectiveness of the procedure among patients with PD and otherwise healthy individuals. According to the results, absolute reduction of VAS and ODI did not differ significantly between cases and controls, although percentage improvement of both measures was significantly lower for the PD group, fact attributed to limited number of cases and higher preoperative values in the parkinsonic group.

Improvement of lower limp symptoms and functional ability due to remission of low back pain is interpreted in a significant improvement in quality of life in those patients. A year after TPED, all aspects of quality of life showed statistically significant improvement from baseline. BP and role physical showed the maximum improvement, followed by RE, PF, $\mathrm{SF}$ and $\mathrm{V} . \mathrm{MH}$, and $\mathrm{GH}$ were subjected to less evident increase. Quality of life following TPED has been previously studied in normal adults with a minimum follow-up period of 2 years and interval measurements at 6 months by Peng et al. ${ }^{25}$. Our results suggest that PD population with lumbar disc hernia is, generally, characterized by lower HRQoL compared to non-PD population at the preoperative setting and within 6 months after surgery, in all aspects measured by SF-36 questionnaire. BP, though, surpassed the respective value for normal population provided by the study of Peng et al. ${ }^{25)}$ at 6 months. A study, conducted by Schroeder et al. ${ }^{31)}$, has investigated the progress of quality of life following open lumbar spine surgery in 96 patients diagnosed with PD. Quality of life was measured by Short Form -12 physical and mental component summary. SF-12 physical component summary improved significantly at the end of a mean follow-up period of 30.1 months, while the SF-12 mental component summary failed to record a similar significant improvement. In our study the SF-36 mental health component showed significant statistical improvement one year after endoscopic discectomy. A possible beneficial impact of endoscopy on the mental aspects of quality of life in those patients, on the contrary to open procedures, should be further investigated.

Percentage improvement of ODI and VAS, as well as absolute increase in the aspects of SF-36 did not differ among males and females or among the different operated levels (L3-4, L4-5, L5-S1). The only exception was percentage improvement of VAS, analyzed according to level of operation. Intervention on L4-L5 level was accompanied by significantly higher percentage improvement in VAS compared to L5-S1 level. Taking into consideration the minimum change of VAS capable to produce clinical difference (which is estimated at $16 \mathrm{~mm})^{7}$, this difference lacks clinical significance. Subsequently, the beneficial effect of TPED in radiculopathy, low back pain and quality of life in parkinsonic population is significant and independent of gender or level of operation, 1 year after surgery.

Taking into consideration the poor outcome of open spine surgery in parkinsonic population ${ }^{2,20,29)}$, the main aggravating factors predicting the need of revision surgery after open procedures, such as the severity of the PD and the comorbidities (diabetes mellitus, osteoporosis) $^{31)}$, as well as the satisfactory clinical outcome of TPED in terms of quality of life, TPED for lumbar disc herniation should be considered as alternative option to traditional procedures, especially in patients with severe PD and other comorbidities.

The strengths of our study arise from the selection of endoscopy (TPED) for the surgical management of lumbar disc hernia in PD patients and not traditional open procedures, that have been previously studied the prospective data collection and homogenous sample in terms of diagnosis and indications for endoscopy, as well as the inclusion of HRQoL in the assessment of clinical outcome. Our study, however, is subjected to some limitations due to (1) the small number of cases (2) the noninclusion of duration and severity of the disease (3) noninclusion of comorbidities and other demographic characteristics that influence health related quality of life and (4) the absence of a control group, consisted of PD patients that would undergo discectomy with open procedures. The latter was not feasible, taking into consideration the prevalence of PD in general population and, especially, in the subgroup diagnosed with lumbar disc hernia, programmed to undergo spine surgery, as well as the absence of any contradiction for TPED, that would lead us to the decision for open discectomy. However, we used the already existing data of TPED on general population to facilitate comparison.

\section{CONCLUSION}

Endoscopic discectomy via the foramen achieves satisfactory improvement in leg pain, low back pain and quality of life in patients suffering from PD. Taking into consideration the disappointing results of open surgery in this specific population, TPED should be considered as an alternative in the management of lumbar herniation in patients with PD. 


\section{CONFLCT OF INTEREST}

No potential conflict of interest relevant to this article was reported.

\section{REFERENCES}

1. Adams MA, Freeman BJ, Morrison HP, Nelson IW, Dolan P: Mechanical initiation of intervertebral disc degeneration. Spine (Phila Pa 1976) 25:1625-1636, 2000

2. Babat LB, McLain RF, Bingaman W, Kalfas I, Young P, RufoSmith C: Spinal surgery in patients with Parkinson's disease: construct failure and progressive deformity. Spine (Phila Pa 1976) 29:2006-2012, 2004

3. Bombardier C: Outcome assessments in the evaluation of treatment of spinal disorders. Introduction. Spine (Phila Pa 1976) 25:3097-3099, 2000

4. Bonnet AM, Houeto JL: Pathophysiology of Parkinson's disease. Biomed Pharmacother 53:117-121, 1999

5. Broetz D, Eichner M, Gasser T, Weller M, Steinbach JP: Radicular and nonradicular back pain in Parkinson's disease: a controlled study. Mov Disord 22:853-856, 2007

6. Choi HJ, Smith JS, Shaffrey CI, Lafage VC, Schwab FJ, Ames $\mathrm{CP}$, et al: Coronal plane spinal malalignment and Parkinson's disease: prevalence and associations with disease severity. Spine J 15:115-121, 2015

7. Copay AG, Glassman SD, Subach BR, Berven S, Schuler TC, Carreon LY: Minimum clinically important difference in lumbar spine surgery patients: a choice of methods using the Oswestry Disability Index, Medical Outcomes Study questionnaire Short Form 36, and pain scales. Spine J 8:968-974, 2008

8. Davidson M, Keating JL: A comparison of five low back disability questionnaires: reliability and responsiveness. Phys Ther 82 : 8-24, 2002

9. de Boer AG, Wijker W, Speelman JD, de Haes JC: Quality of life in patients with Parkinson's disease: development of a questionnaire. J Neurol Neurosurg Psychiatry 61:70-74, 1996

10. Doherty KM, van de Warrenburg BP, Peralta MC, Silveira-Moriyama L, Azulay JP, Gershanik OS, et al: Postural deformities in Parkinson's disease. Lancet Neurol 10:538-549, 2011

11. Etchepare F, Rozenberg S, Mirault T, Bonnet AM, Lecorre C, Agid Y, et al: Back problems in Parkinson's disease: an underestimated problem. Joint Bone Spine 73:298-302, 2006

12. Fairbank JC, Pynsent PB: The Oswestry Disability Index. Spine (Phila Pa 1976) 25:2940-2952, 2000

13. Ford B: Pain in Parkinson's disease. Mov Disord 25 Suppl 1: S98-103, 2010

14. Goetz CG, Tanner CM, Levy M, Wilson RS, Garron DC: Pain in Parkinson's disease. Mov Disord 1(1):45-9, 1986

15. Guilfoyle MR, Seeley H, Laing RJ: The Short Form 36 health survey in spine disease: validation against condition-specific measures. Br J Neurosurg 23:401-405, 2009

16. Hughes AJ, Daniel SE, Blankson S, Lees AJ: A clinicopathologic study of 100 cases of Parkinson's disease. Arch Neurol 50:140148, 1993

17. Kambin P, Brager MD: Percutaneous posterolateral discectomy.
Anatomy and mechanism. Clin Orthop Relat Res (223):145-154, 1987

18. Kapetanakis S, Giovannopoulou E, Charitoudis G, Kazakos K: Transforaminal percutaneous endoscopic discectomy for lumbar disc herniation in Parkinson's disease: A Case-Control Study. Asian Spine J 10:671-677, 2016

19. Kawakami M, Tamaki T, Ando M, Yamada H, Hashizume H, Yoshida M: Lumbar sagittal balance influences the clinical outcome after decompression and posterolateral spinal fusion for degenerative lumbar spondylolisthesis. Spine (Phila Pa 1976) 27: 59-64, 2002

20. Koller H, Acosta F, Zenner J, Ferraris L, Hitzl W, Meier O, et al: Spinal surgery in patients with Parkinson's disease: experienc es with the challenges posed by sagittal imbalance and the Parkinson's spine. Eur Spine J 19:1785-1794, 2010

21. Mandir AS, Vaughan C: Pathophysiology of Parkinson's disease. Int Rev Psychiat 12:270-280, 2000

22. Mayer HM, Brock M: Percutaneous endoscopic lumbar discecto my (PELD). Neurosurg Rev 16:115-120, 1993

23. Nellensteijn J, Ostelo R, Bartels R, Peul W, van Royen B, van Tulder M: Transforaminal endoscopic surgery for symptomatic lumbar disc herniations: a systematic review of the literature. Eur Spine J 19:181-204, 2010

24. Oh JK, Smith JS, Shaffrey CI, Lafage V, Schwab F, Ames CP, et al: Sagittal spinopelvic malalignment in Parkinson disease: prevalence and associations with disease severity. Spine (Phila Pa 1976) 39:E833-841, 2014

25. Peng CW, Yeo W, Tan SB: Percutaneous endoscopic lumbar discectomy: clinical and quality of life outcomes with a minimum 2 year follow-up. J Orthop Surg Res 4:20, 2009

26. Peto V, Jenkinson C, Fitzpatrick R, Greenhall R: The development and validation of a short measure of functioning and well being for individuals with Parkinson's disease. Qual Life Res 4:241-248, 1995

27. Rubenstein LM, Voelker MD, Chrischilles EA, Glenn DC, Wallace RB, Rodnitzky RL: The usefulness of the Functional Status Questionnaire and Medical Outcomes Study Short Form in Parkinson's disease research. Qual Life Res 7:279-290, 1998.

28. Ruetten S, Komp M, Merk H, Godolias G: Full-endoscopic interlaminar and transforaminal lumbar discectomy versus conventional microsurgical technique: a prospective, randomized, controlled study. Spine (Phila Pa 1976) 33:931-939, 2008

29. Sapkas G, Lykomitros V, Soultanis K, Papadopoulos EC, Papadakis M: Spinal surgery in patients with Parkinson's disease: unsatisfactory results, failure and disappointment. Open Orthop J 8:264-267, 2014

30. Schrag A, Jahanshahi M, Quinn N: How does Parkinson's disease affect quality of life? A comparison with quality of life in the general population. Mov Disord 15:1112-1118, 2000

31. Schroeder JE, Hughes A, Sama A, Weinstein J, Kaplan L, Cammisa FP, et al. Lumbar spine surgery in patients with Parkinson disease. J Bone Joint Surg Am 97:1661-1666, 2015

32. Twelves D, Perkins KS, Counsell C: Systematic review of incidence studies of Parkinson's disease. Mov Disord 18:19-31, 2003

33. Valkovic P, Minar M, Singliarova H, Harsany J, Hanakova M, Martinkova J, et al: Pain in Parkinson's disease: a cross-sectional study of its prevalence, types, and relationship to depression and quality of life. PLoS One 10:e136541, 2015 
34. von Campenhausen S, Bornschein B, Wick R, Bötzel K, Sampaio C, Poewe W, et al: Prevalence and incidence of Parkinson's disease in Europe. Eur Neuropsychopharmacol 15:473-490, 2005

35. Ware JE Jr, Bjorner JB, Kosinski M: Practical implications of item response theory and computerized adaptive testing: a brief summary of ongoing studies of widely used headache impact scales. Med Care 38(9 Suppl):II73-82, 2000

36. Ware JE: SF-36 Health Survey: Manual and Interpretation Guide.
Lincoln (RI): QualityMetric Inc., 2000

37. Wewers ME, Lowe NK: A critical review of visual analogue scales in the measurement of clinical phenomena. Res Nurs Health 13:227-236, 1990

38. Zanoli G, Jönsson B, Strömqvist B: SF-36 scores in degenerative lumbar spine disorders: analysis of prospective data from 451 patients. Acta Orthop 77:298-306, 2006 